\title{
Photothermal high-speed crystal actuation and the simulation
}

\author{
S. Hasebe ${ }^{1}$, Y. Hagiwara' ${ }^{1}$, J. Komiya ${ }^{1}$, M. Ryu², H. Fujisawa ${ }^{3}$, J. Morikawa ${ }^{3}$, T. Katayama ${ }^{4}$, D. Yamanaka ${ }^{4}$, \\ A. Furube ${ }^{4}$, H. Sato ${ }^{5}$, T. Asahi ${ }^{1,6}$, H. Koshima ${ }^{6}$
}

1 Graduate School of Advanced Science and Engineering, Waseda University, 3-4-1 Okubo, Shinjuku-ku, Tokyo 169-8555, Japan ${ }^{2}$ Research Institute for Material and Chemical Measurement, National Metrology Institute of Japan (AIST), Tsukuba Central 3, 1-1-1 Umezono, Tsukuba 305-8563, Japan,

${ }^{3}$ School of Materials and Chemical Technology, Tokyo Institute of Technology, Ookayama, Meguro-ku, Tokyo 152-8550, Japan

${ }^{4}$ Department of Optical Science, Tokushima University, 2-1 Minamisanjyojima-cho, Tokushima-shi 770-8506, Japan,

${ }_{5}^{5}$ Rigaku Corporation, 3-9-12 Matasubara-cho, Akishima-shi, Tokyo 196-8666, Japan, 6Research Organization for Nano and Life Innovation, Waseda University, 513 Wasedatsurumaki-cho, Shinjuku-ku, Tokyo 162-0041, Japan

$$
\text { jenepaletemps@suou.waseda.jp }
$$

Mechanically responsive organic materials have attracted attention from perspective of both basic research and applications in smart actuators and soft robots [1]. We have developed many mechanical crystals such as azobenzene [2] and salicylidenealine [3], mainly based on photoisomerization. However, photoisomerization has some disadvantages for crystal actuation, such as a limited number of photoisomerizable crystals, slow actuation speed, and no actuation of thick crystals. Here we report photothermally driven fastbending actuation and simulation of a salicylideneaniline derivative crystal with an $o$-amino substituent in enol form (enol-1).

$\mathrm{X}$-ray crystallographic analysis revealed that enol-1 crystal belonged to the space group, $P 2_{1}$, showing that the enol-1 molecule is achiral but forms chiral crystal. The molecules were connected weakly through the intermolecular hydrogen bond chains in a two-fold helical manner to form the herringbone structure along the $b$ axis (Figure 1a, b). Absorption spectra of a thin enol-1 crystal revealed that enol-1 crystal exhibited fast photoisomerization from enol to trans-keto form ( $\tau=0.9 \mathrm{~s})$ by UV light and fast back-isomerization $(\tau=4.2 \mathrm{~s})$ from trans-keto to enol form.

Under UV light irradiation, the thin $(<20 \mu \mathrm{m})$ crystals bent away from the light source quickly (in a few seconds) by photoisomerization. In contrast, the thick $(>20 \mu \mathrm{m})$ crystals bent very quickly (in several milliseconds) due to the photothermal effect, finally achieving $500-\mathrm{Hz}$ high-frequency bending by pulsed UV laser irradiation. We propose a possible mechanism in which photothermally driven bending is caused by a non-steady temperature gradient in the thickness direction. The temperature gradient was calculated based on a one-dimensional non-steady heat conduction equation, resulting in the successful simulation of bending via the photothermal effect and the elucidation of the proposed mechanism (Figure 1c). Most materials that absorb light show their own photo-thermal effects. The creation of crystal motion via the photothermal effect will expand the designability and versatility of mechanical crystals in the future.
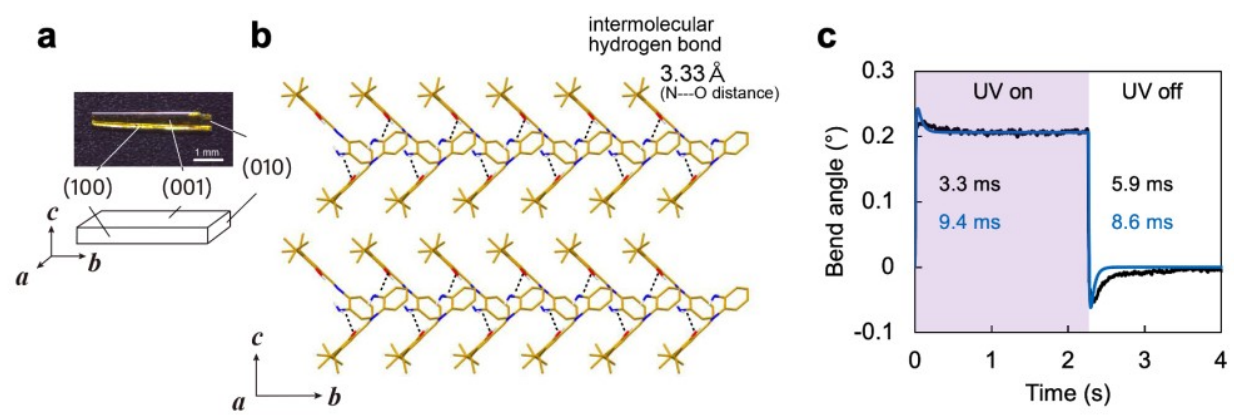

Figure 1. (a) Face indices of enol-1 crystal. (b) Two-fold helical arrays formed via intermolecular hydrogen chain along the $b$ axis on the (100) side face. (c) Time dependence of measured (black) and simulated (blue) bend angle. The numbers indicate time constants.

[1] Koshima, H. (2020). Mechanically Responsive Materials for Soft Robotics, ed. H. Koshima, Wiley-VCH, Weinheim.

[2] Koshima, H., Ojima, N., Uchimoto, H. (2009). J. Am. Chem. Soc. 131, 6890-6891.

[3] Koshima, H., Takechi, K., Uchimoto, H., Shiro, M., Hashizume, D. (2011). Chem. Commun. 47, 11423-11425.

Keywords: photomechanical crystal; photoisomerization; photothermal effect; heat conduction; salicylideneaniline 